\title{
The urgency-gating model can explain the effects of early evidence
}

\author{
Matthew A. Carland ${ }^{1} \cdot$ David Thura $^{1} \cdot$ Paul Cisek $^{1}$
}

Published online: 24 September 2015

(C) Psychonomic Society, Inc. 2015

\begin{abstract}
In a recent report, Winkel, Keuken, van Maanen, Wagenmakers \& Forstmann (Psychonomics Bulletin and Review 21(3): 777-784, 2014) show that during a random-dot motion discrimination task, early differences in motion evidence can influence reaction times (RTs) and error rates in human subjects. They use this as an argument in favor of the drift-diffusion model and against the urgency-gating model. However, their implementation of the urgency-gating model is incomplete, as it lacks the low-pass filter that is necessary to deal with noisy input such as the motion signal used in their experimental task. Furthermore, by focusing analyses solely on comparison of mean RTs they overestimate how long early information influences individual trials. Here, we show that if the urgency-gating model is correctly implemented, including a low-pass filter with a $250 \mathrm{~ms}$ time constant, it can successfully reproduce the results of the Winkel et al. experiment.
\end{abstract}

Keywords Decision making $\cdot$ Response time models · Computational modeling $\cdot$ Drift-diffusion model $\cdot$ Perceptual discrimination

\section{Introduction}

Many current models of decision-making (Bogacz et al., 2006; Busemeyer \& Townsend, 1993; Gold \& Shadlen,

This paper is a commentary on Winkel et al., 2014; doi:10.3758/s13423013-0551-8.

Paul Cisek

paul.cisek@umontreal.ca

1 Groupe de Recherche sur le Système Nerveux Central (GRSNC), Department of Neurosciences, Université de Montréal, C.P. 6128 Succursale Centre-ville, Montréal H3C 3J7, QC, Canada
2007; Ratcliff, 1978; Usher \& McClelland, 2001) suggest that during deliberation, the brain integrates evidence in favor of each choice until the total integrated evidence reaches a threshold, the setting of which determines an accuracy criterion. The most influential of these is called the "drift-diffusion model" (DDM) (Ratcliff, 1978), and has been used to explain reaction times (RTs) and error rates as well as neural activity in a wide variety of decision-making tasks (Churchland, Kiani, \& Shadlen, 2008; Domenech \& Dreher, 2010; Gold \& Shadlen, 2007; Heekeren, Marrett, \& Ungerleider, 2008; Palmer, Huk, \& Shadlen, 2005; Ratcliff et al., 2007; Roitman \& Shadlen, 2002). One feature of the DDM is that, like any integrator, it is robust in the presence of noise.

However, in most natural situations the environment can change rapidly, and animals must be able to respond to such changes quickly. Integrators are not ideal in this regard because in order to change an ongoing decision they must first reverse the previously integrated evidence for the old choice. In contrast, a low-pass filter with a short time constant can respond more quickly to changes in sensory evidence while remaining robust to noise. For this reason, we and others have suggested that sensory evidence is not integrated continuously but rather low-pass filtered and combined with an independent signal related to the urgency for making a choice - and that together these bring neural activity to a decision threshold (Cisek, Puskas, \& El-Murr, 2009; Ditterich, 2006; Thura et al., 2012). We have called this the "urgency-gating model" (UGM).

Importantly, nearly all of the experiments typically cited in support of the drift-diffusion model have used tasks in which subjects view stimuli in which the informational content pertinent to the decision is held constant throughout each trial. For random-dot motion tasks this means that the underlying direction and degree of coherent motion are constant and always present during any given trial. However, if evidence is held constant in this way, no conclusive distinction can be 
made between the DDM and the UGM because under such conditions both of these models make very similar predictions that are difficult to distinguish experimentally. Thus, the best way to distinguish these models is to design tasks in which the evidence changes within trials - a scenario for which the models make qualitatively different predictions.

Specifically, because it keeps a running sum of all sensory evidence, the DDM predicts that adding or removing sensory evidence at any point prior to the decision will affect the time at which the integrated sum of evidence crosses the threshold, thereby affecting the response time. In contrast, the UGM posits that evidence is computed quickly using a low-pass filter with a fairly short time constant. Thus, it more quickly tracks the current state of evidence while filtering out spurious, high-frequency noise (Fig. 1a, b). This evidence-tracking signal is subsequently combined with a separate "urgency signal" that independently grows over time to bring the resulting neural activity to a decision threshold (Fig. 1c, d), as commonly observed in physiological studies (Churchland, Kiani, \& Shadlen, 2008; Gluth, Rieskamp, \& Buchel, 2012; Thura \& Cisek, 2014). Because the strength of the evidence signal is dependent primarily on the recent state of the evidence at any given time, early transient increases in stimulus strength will "leak out" later in the trial.

Importantly, the amount of time it takes for the evidence signal to transition from representing one evidence level to the next is determined by the time constant of the low-pass filter. By this logic, any change in the level of stimulus strength should only affect response times for trials in which the decision was made within a limited temporal window of this change in evidence strength (i.e., before the prior evidence has fully leaked away). Decisions made past this critical window ought to be indistinguishable from trials in which evidence was held constant (see Fig. 1d). We have shown support for this latter prediction in recent experiments, first using a task in which subjects made decisions based on discrete events (Cisek et al., 2009), and later using a variation of the randomdot task in which the motion coherence was changing during each trial (Thura et al., 2012). We have also shown that while monkeys make decisions about changing sensory information, neural activity in motor regions tracks evidence quickly (with old evidence leaking out within $200 \mathrm{~ms}$ ), and combines it with a growing urgency signal (Thura \& Cisek, 2014).

In a recent paper, Winkel et al. (2014) also used a changingevidence task but reached the opposite conclusion with respect to the two models. In their task, subjects viewed random-dot motion in which the signal early in the trial varied briefly between trials in terms of direction and duration, and these brief early variations significantly influenced the mean RT of subjects in a stimulus-dependent manner, similar to earlier studies of motion pulses (Huk \& Shadlen 2005; Kiani, Hanks, \& Shadlen 2008). They then attempted to model and replicate their behavioral results using the two models
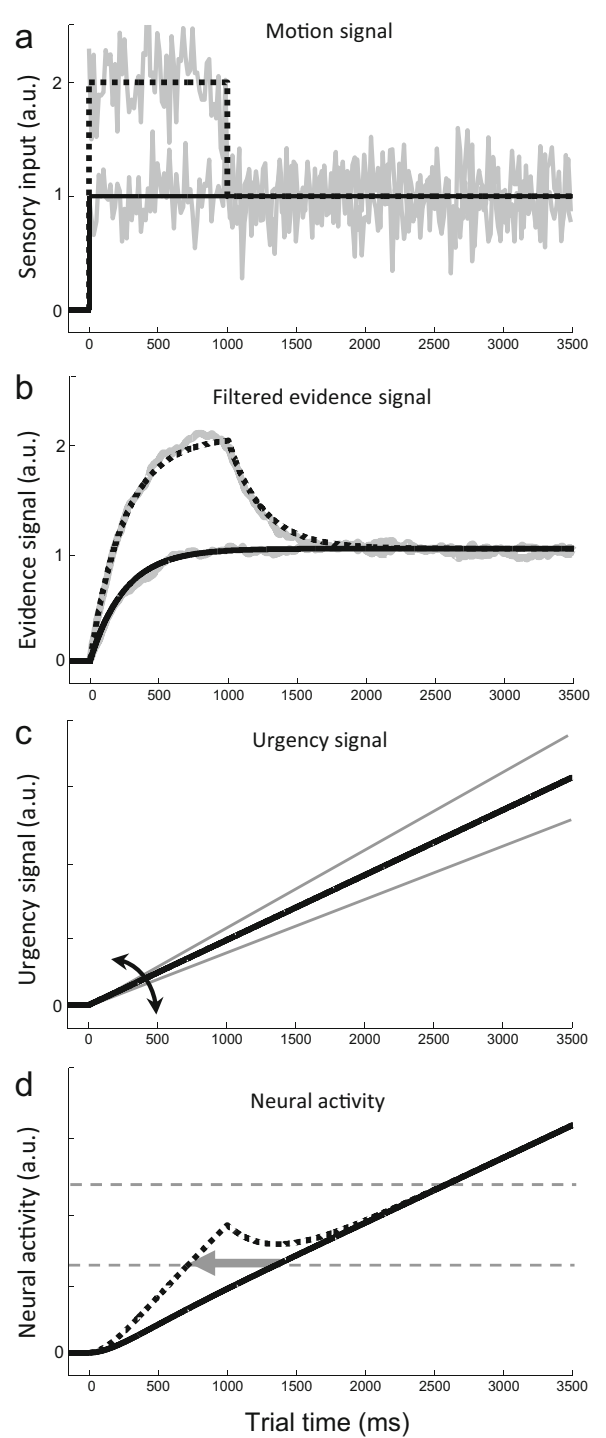

Fig. 1 (a) Example of a simple step input with- and without an early increase in evidence ("pulse") $1000 \mathrm{~ms}$ in length, during which the signal strength is doubled. Black lines represent the underlying motion signal in each condition, whereas the grey traces portray the resulting sensory input which is subject to noise (SNR $=4: 1$ ). (b) The same evidence signal depicted in (a), passed through a low-pass filter with a time constant of $250 \mathrm{~ms}$. Black lines depict the filter's response to a pure signal without noise, as per the black traces in (a); grey lines show the filter's response to the same noisy signal depicted in (a). (c) An independent "urgency" signal that increases over the course of a decision. The urgency signal's mean slope (black line) can vary across tasks, enabling different decisionmaking strategies for different contexts. Additionally, the urgency signal slope is itself subject to inter-trial variations (grey lines) which generate variability in decision times even for identical trials within a given task context. (d) The combined result of the filtered evidence (b) and urgency signal (c), resulting in rise-to-threshold neural activity. The dashed grey lines depict two different threshold settings. Note that this model predicts an effect of early evidence on decision time only for a certain time window following early evidence; decisions made sufficiently later will no longer show an effect and will be indistinguishable from reaction times (RTs) from trials in which the evidence was constant. The length of the time window for early evidence's efficacy is effectively determined by the time constant of the filter (i.e., the leak parameter), as well as the slope of the urgency signal within a given trial 
described above. In so doing they obtained qualitatively different results for each: the DDM showed a pronounced sensitivity to early changes in evidence strength - even for decisions taken relatively late in a trial - whereas their implementation of the UGM was irresponsive to these early changes. Because the behavioral data revealed that human decisionmakers are sensitive to early evidence, it led them to conclude that the DDM was better-suited to explain behavior in such tasks. However, Winkel et al. did not consider two important points. The first relates to their implementation of the urgencygating model and the second pertains to how they analyzed and interpreted their data.

\section{The urgency-gating model includes a low-pass filter}

The UGM includes a low-pass filter to reduce high-frequency noise in the stimulus while remaining sensitive to the lowfrequency signal. This is important, especially if one hopes to simulate behavior in a task like random-dot motion discrimination in which the stimulus is highly noisy. However, Winkel et al. chose to use a "UGM without temporal filtering" (their Eq. 2).

In the original UGM paper (Cisek et al. 2009), we compared four versions of evidence integrator models and two versions of the urgency-gating model, one without ("model 5 ") and one with a low-pass filter ("model 6"). The former was meant solely as an illustrative step, and we emphasized the importance of low-pass filtering in the original 2009 paper, as well as in subsequent publications (Thura et al., 2012; Thura \& Cisek, 2014; Thura, Cos, Trung \& Cisek, 2014). Unfortunately, it seems this caused some confusion. In particular, in the behavioral task described in Cisek et al. (2009) there was no noise so both models 5 and 6 behaved similarly. It is perhaps for that reason that Winkel et al. assumed that low-pass filtering is not needed in general, and did not try including it in their simulations. This is unfortunate because, as will be shown below, if the UGM is implemented correctly and includes a low-pass filter, then it easily captures the results of their experiment.

Here, we aim to clarify why the filter is necessary and how it relates to concepts such as "leaky integration" (Usher \& McClelland, 2001). Consider a general implementation of a leaky integrator described by the following first-order linear differential equation

$\frac{d x}{d t}=-L x+g E$

Here, $x$ is the neural decision variable, $g$ is a gain factor, and $L$ is a leak parameter. The sensory evidence is denoted as $E(t)$, and we assume that it is subject to Gaussian noise with mean zero, standard deviation $\sigma$, and frequency $F$.
Now consider how changes in these parameters influence the dynamics of the system. If the leak is zero, then $x$ simply integrates $E$ with gain $g$. In other words, it becomes equivalent to the DDM. If the leak parameter is non-zero and positive, then the system behaves like a low-pass filter with a time constant equal to $1 / L$ and gain equal to $g / L$. This can be demonstrated by rewriting Eq. (1) as

$\tau \frac{d x}{d t}=-x+\frac{g}{L} E$

where the time constant is $\tau=1 / L$. See Fig. $1 \mathrm{a}$ and $\mathrm{b}$ for an example of how $x$ responds to a simple step input buried in noise (mean 0 , standard deviation 0.25 ). Importantly, the lowpass filter is just as effective in removing high-frequency noise as a pure integrator.

Now consider what happens in the limit as both $L$ and $g$ go to infinity. The result is that the time constant becomes zero so the left-hand side of Eq. (2) goes to zero, leaving

$x=h E$

where $h$ is a gain equal to $g / L$. This is effectively what was implemented by Winkel et al. in their equations. Consequently, here we will refer to their model as a "zero time constant" version of the UGM. This is functionally and mathematically equivalent to the wholesale removal of the low-pass filter component of the model, and thus renders it entirely unable to handle noise.

The differences between the UGM with- and without a low-pass filter are illustrated in Fig. 2. Note that when presented with constant, noiseless evidence, the models behave very similarly. There is a slight difference in the predicted build-up of neural activity (compare solid and dashed black lines) but we believe this is unlikely to be empirically distinguishable. However, the presence of a filter nonetheless has strong consequences when dealing with noise. Specifically, the filtered version (with a time constant of $250 \mathrm{~ms}$; solid grey line) is much less susceptible to noise than the unfiltered version (dashed grey line).

Note that in both the filtered and unfiltered versions, nearly all of the activity build-up over time can be attributed to the growth of the urgency signal, rather than the integration of evidence over time. Thus, the UGM's primary point of departure from the DDM is not in whether activity builds-up over time, but in what drives such activity build-up. The urgency signal replaces evidence integration as an explanation for the build-up, but some estimate of evidence is still necessary. That estimate is provided by a low-pass filter with a short time constant, as opposed to the infinite (or very large) time constant assumed by the DDM.

In summary, filtering is of central importance to the operation of the UGM and cannot be omitted from any fair comparison of model performance. This raises a question: If the 


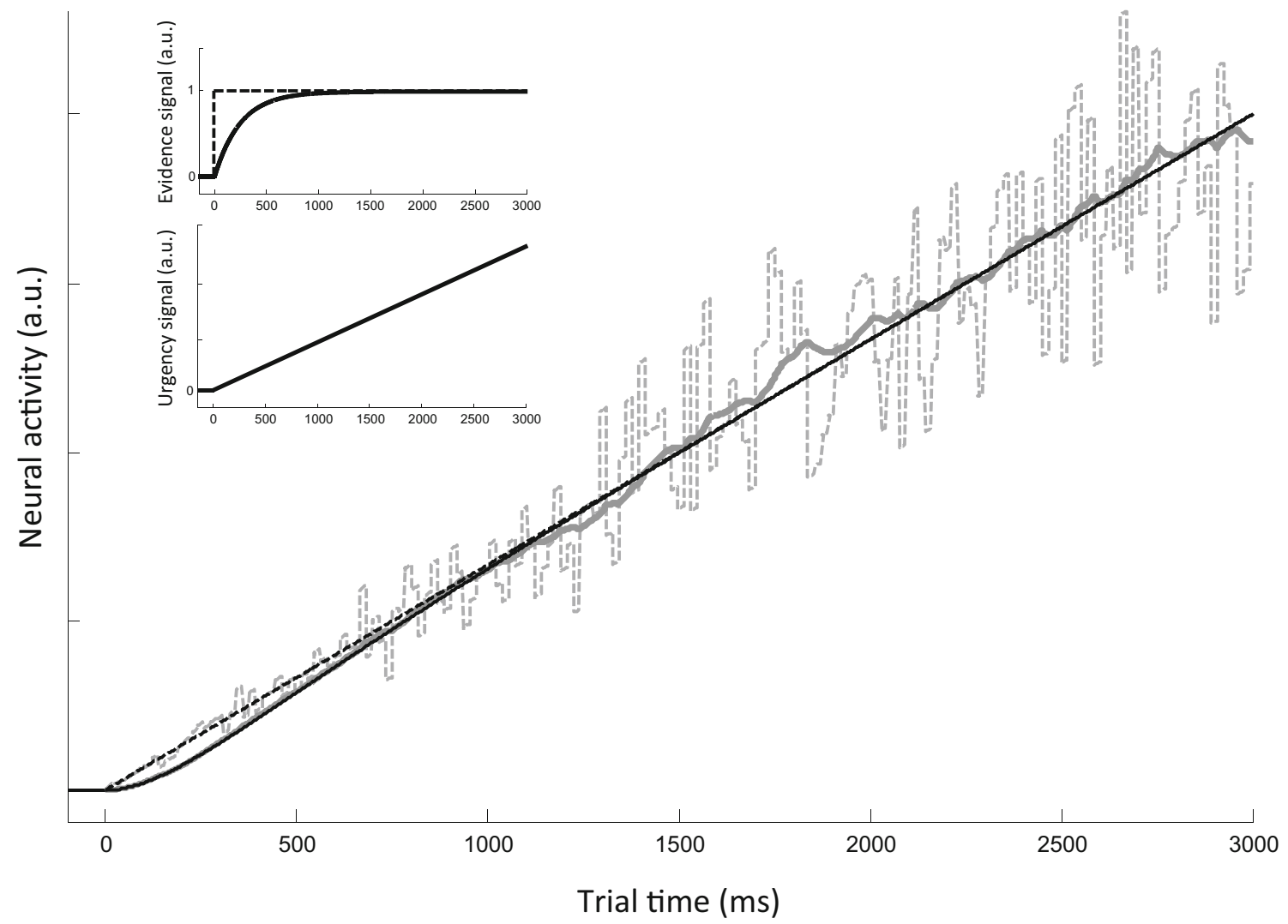

Fig. 2 Neural activity predicted by the UGM in response to a step input signal, assuming a filter with a $250-\mathrm{ms}$ time constant (solid lines) versus a version without a filter (dashed lines) in response to either a constant noiseless sensory input (black) or input with noise that is six times

UGM were to be implemented correctly, could it reproduce the results reported by Winkel et al.? And, if so, what timeconstant would be necessary?

\section{Simulating the data of Winkel et al.}

Using Eq. (1) to implement the low-pass filter, we multiply the resulting filtered evidence signal by a growing urgency signal:

$y(t)=x(t) \cdot U(t)$

where $U(t)=(b+m t) \eta$ and $\eta$ is inter-trial noise (i.e., variability in the rate of growth of the urgency signal that changes from trial to trial, but not within a trial). The decision is made when $y(t)$ reaches a threshold $T$ and we add a constant "non-decision time" $t_{0}$ to obtain the total RT $\left(R T=t+t_{0}\right)$. Note: in our original description of the UGM (Cisek et al. 2009) we implied that inter-trial variability influenced the evidence term, while here we imply that it influences the urgency signal. While this does not strictly make any difference because the terms are multiplied, we speculate that at the neural level, the more likely scenario is that inter-trial variability influences the urgency signal.

Instead of using a minimization procedure to find the parameter values that produced the best fits to the full data set, stronger than the signal (grey). Note that the presence or absence of a filter has only a small influence on the time course of the average overall build-up of neural activity, but a major effect on the attenuation of noise. Insets illustrate the estimated evidence (top) and the urgency signal (bottom)

we fixed as many of our parameter settings as possible using some of our own data from humans performing the randomdot motion task without any changes in motion. We obtained an estimate for the non-decision delay (NDD $=481 \mathrm{~ms}$ ) by averaging the mean RTs from all subjects from "easy" trials in which motion was held constant at $50 \%$ coherence, allowing for very rapid discrimination of motion direction (admittedly, this is probably an over-estimate, as even a strong motion signal of $50 \%$ coherence will still require some perceptual processing). We then estimated additional parameter values on the basis of prior UGM fits to data obtained from trials in which motion coherence was held constant at $3 \%$. On this basis we assumed a fairly large signal noise (Gaussian distribution with $\mu=0$ and $\sigma=6, \mathrm{SNR}=1: 6)$ with a frequency limited by the refresh rate of the stimulus display $(60 \mathrm{~Hz})$.

We fit the remaining parameters as follows. The signal gain ( $g=0.03)$, leak ( $L=0.004$, corresponding to a time constant of $250 \mathrm{~ms})$, decision threshold $(T=16300)$, and inter-trial noise (log-normal distribution with $\mu=0.7$ and $\sigma=0.2$ ) were chosen to produce a simulated reaction-time distribution whose mean was as close as possible to that reported for condition \#9 in Winkel et al. (i.e., the closest experimental analogue to our own "difficult" $3 \%$ coherence, constant-evidence trials). The remaining eight trial conditions were then simulated using the 
description provided by Winkel et al., as shown in our Fig. 3 . Our results are shown in Fig. $4 a$ and $b$, which compare the output of our version of the UGM to the data reported by Winkel et al. as well as to their simulations of the DDM and their "zero time constant" version of the UGM.

Clearly, when it is implemented correctly, the UGM simulates the behavioral data quite well, with RTs for the six trial types containing the most evidence lying within the standard error of the mean for most data points (Fig. 4a), and with corresponding error rates matching those of the behavioral data reported by Winkel et al. (Fig. 4b). More importantly, over and beyond any quantitative aspects of the models' fits is the UGM's qualitatively-demonstrated sensitivity to early evidence. While the extent of this influence of early evidence is weaker in our model than in the behavioral data (as reflected in the overall slope of the data points in Fig. 4a and the longer RTs in trial types 1-3), it is nonetheless quite robust (as evidenced by the small error margins). This places it in stark contrast to Winkel et al.'s "zero time constant" version of the UGM, which showed no effect of early evidence at all - an observation which in part motivated their dismissal of the UGM. Of course, neither of these models should be expected to provide a perfect fit, because they don't attempt to capture all the complexities of human behavior (e.g., lapses of attention, etc.). Crucially, however, their respective disparities with respect to the present data do not permit a strong ruling in favor of one over the other, as previously reported.

As noted above, a low-pass filter is a leaky integrator, and as such is related to the leaky competing accumulator (LCA) model of Usher and McClelland (2001). However, it is important to recognize how strong the leak that we're proposing really is. With a $250-\mathrm{ms}$ time constant the filter equilibrates to within $15 \%$ of its asymptote in about $500 \mathrm{~ms}$. In the presence of noise, at this point it no longer continues to consistently grow but rather fluctuates around an asymptotic value (see Fig. 1b). This means that after that point, if the threshold is constant, then the only way the system can cross it is through random momentary fluctuations due entirely to noise. Allowing for a $\sim 450$-ms non-decision delay, this would mean that all RTs longer than $950 \mathrm{~ms}$ ( $97 \%$ of the data of Winkel et al.) would be determined purely by random fluctuations. Furthermore, neural activity up to that point would be a saturating exponential function, not the linear build-up often observed ( $c f$. Roitman \& Shadlen, 2002). Instead, if one posits that an urgency signal helps to bring neural activity to the decision threshold, then the result is the UGM - a low-pass

\section{Trial types}
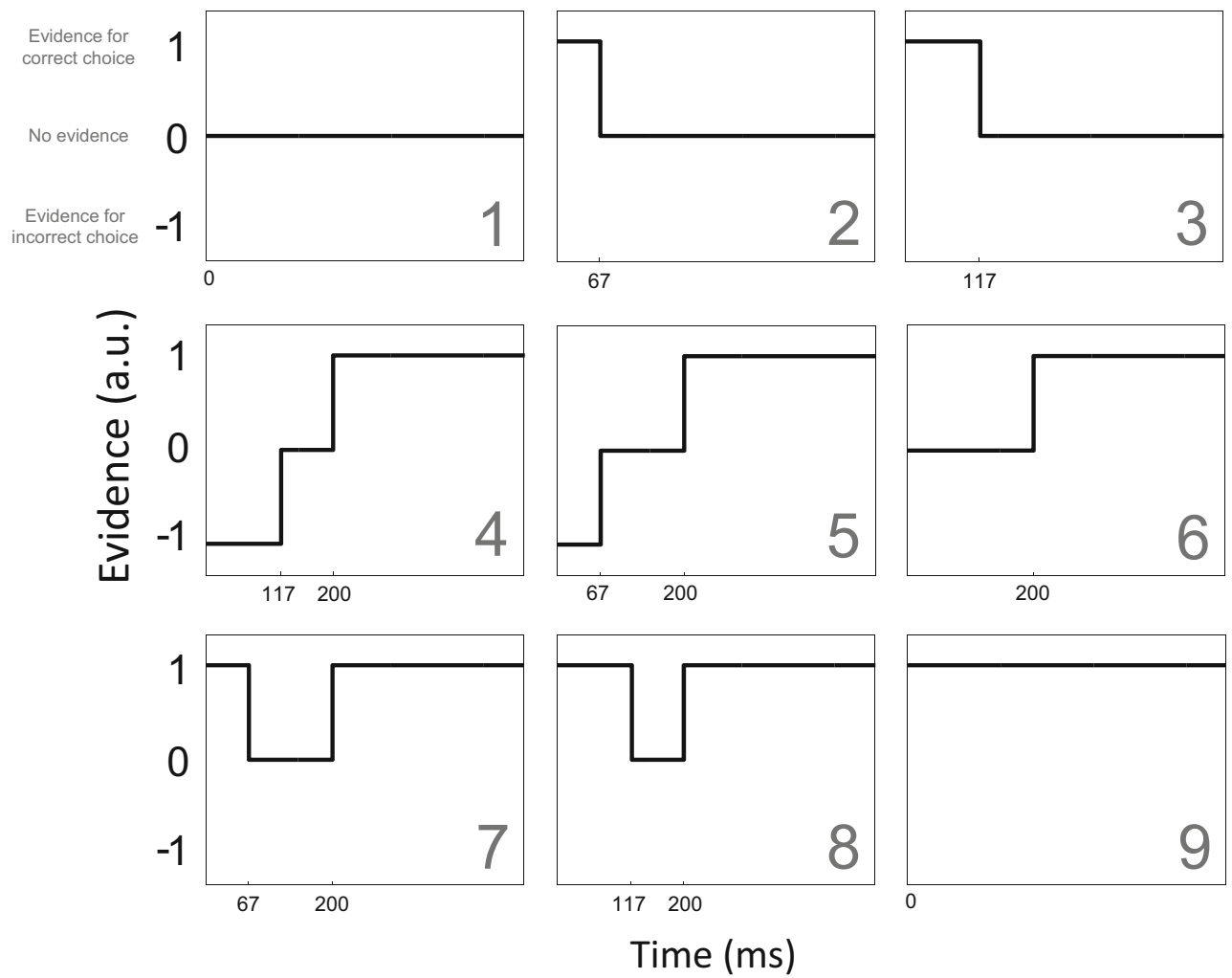

Fig. 3 Trial-type definitions for each of the nine conditions used by Winkel et al. (2014). Exact evidence strength values were not reported by Winkel et al. and are presented here in arbitrary units. At any given time, evidence indicates either the correct target (" 1 "), the incorrect target

("- 1 "), or is absent entirely ("0"), favoring no target. In all cases, evidence values remain constant at their final level for the entire duration of the trial (i.e., up to $2000 \mathrm{~ms}$ ) 


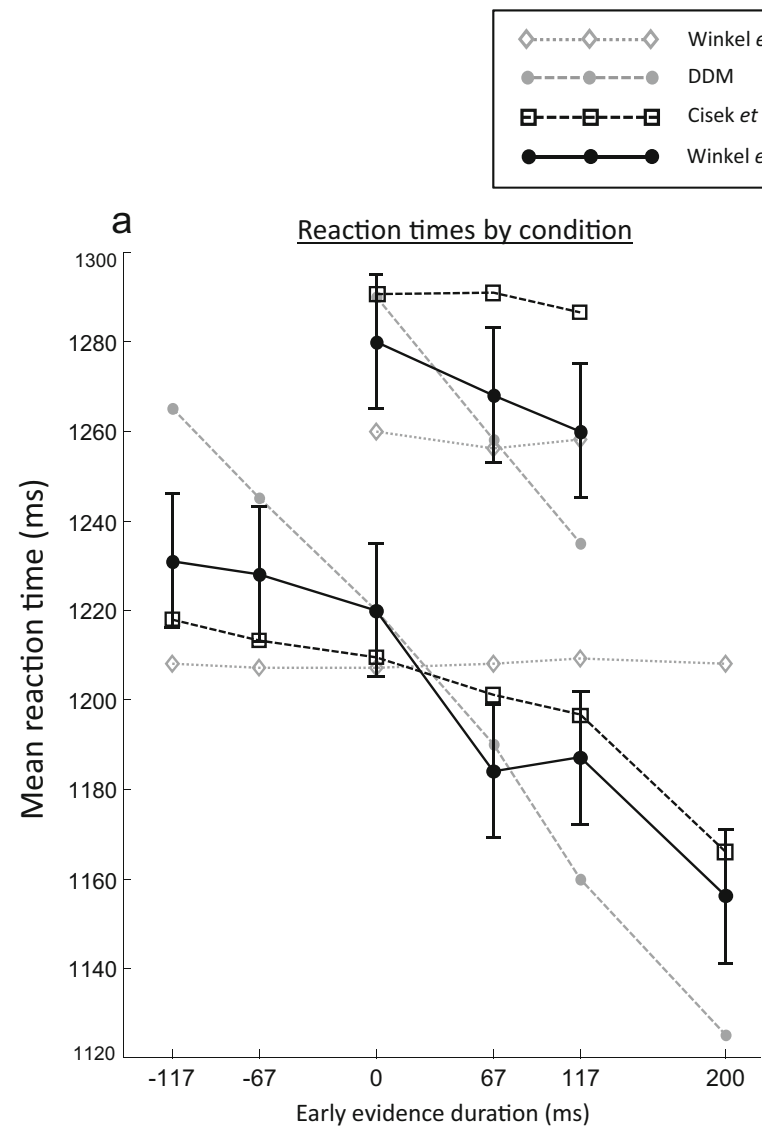

Fig. 4 (a) Means and standard errors for reaction-time (RT) distributions generated by the different models under each task condition (all data points were approximated as faithfully as possible from Winkel et al., 2014; numbered data points refer to the numbered trial types presented in Fig. 3). The "zero time constant" UGM as implemented by Winkel et al. (dotted grey line) shows no sensitivity to early evidence - the main observation on which the authors based their conclusions. When implemented correctly, however, the complete UGM (dashed black line) does capture the overall trends present in the human behavioral

filter with time-varying gain that can explain existing neural and behavioral data (Ditterich, 2006). In summary, a UGM is defined as consisting of two key elements: (1) fast processing of sensory information using a low-pass filter with a relatively short time constant; and (2) an urgency signal that brings the result toward a decision threshold. The specific setting of the time constant is difficult to estimate in behavioral experiments, although prior studies and our recent neural data suggest that in some conditions it can be as short as $100 \mathrm{~ms}$ (Ludwig et al., 2005; Ghose, 2006; Thura \& Cisek, 2014). It could also be task dependent and adjustable (Ossmy et al., 2013).

\section{Interpreting model fits to data}

In the Winkel et al. paper, early motion evidence presented in the first $67-200 \mathrm{~ms}$ was interpreted as "[...having] pronounced

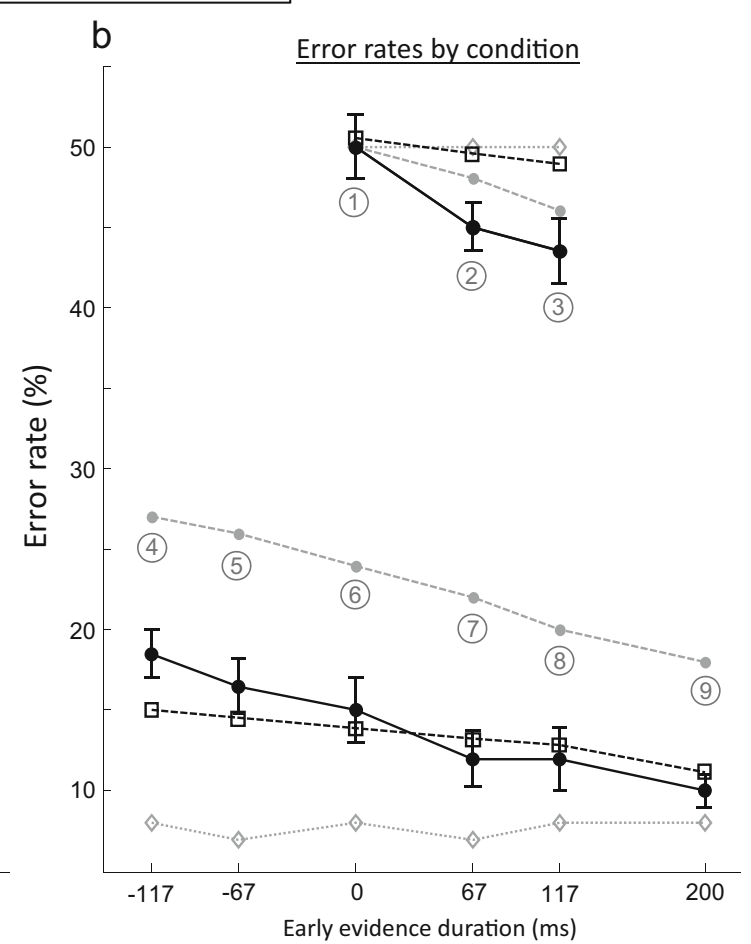

data (solid grey line). Each data point for our implementation of the UGM reflects 50,000 simulated trials; the range of the standard error of the means (SEM) is smaller than the markers themselves and thus has been omitted from both Figs. 4a and b. (b) Error rates generated by each model for each task condition. Winkel et al.'s implementation of the UGM (dotted grey line) is once again insensitive to early evidence. Including the low-pass filter of the UGM, however, does in fact render it responsive to changes in early evidence, and, overall, matches the behavioral data very well

effects $1100 \mathrm{~ms}$ later" on the basis of the resulting changes in the means of the RT distributions. Consequently, on the surface the behavioral data would seem to support a leak-less model such as the DDM, and therefore appear to contradict any model with substantial leak, including our version of the UGM (with a filter time constant of $250 \mathrm{~ms}$ ). However, it is important to bear in mind that the mean of a distribution can be influenced in any number of ways, ranging from an identical systematic bias in every member of that distribution, to a small number of large biases in only a subset of that distribution. This point is of crucial importance when comparing the performance of the two models because examining solely the group mean is not sufficient to determine what kind of change has taken place in the full distribution from which the mean RT is calculated.

To better illustrate these differences, it helps to scrutinize in detail how each model generates variability in RTs in the simplest case of identical constant-evidence trials. It is wellknown that RT distributions in decision-making tasks are very 
broad, and this can be explained either with large intra-trial noise - as in the DDM - or with large inter-trial variability - as in the LATER model (Carpenter \& Williams, 1995) and the UGM (Cisek, Puskas, \& El-Murr, 2009; Thura et al., 2012). Importantly, variability in the urgency signal also provides an alternative explanation for the shift in mean RT distributions reported by Winkel et al. - namely, when the urgency signal is steep, decisions will sometimes be made before the early information has fully leaked out, resulting in a shift in the RT only for those trials. When the urgency signal is shallower, the total decision-related activity will not reach threshold until after the early information has fully leaked out, thereby making the RT indistinguishable from a trial in which no early evidence was presented at all.

In short, the early information may not have lingering effects $1100 \mathrm{~ms}$ later within individual trials. Instead, individual response times are only affected by early evidence in a subset of trials in which the decision was made within a certain time window of the change in evidence. Other trials, in which the decision was made later, will otherwise show no difference in response time because any early evidence will have leaked out by the time the decision is made. Crucially, however, the mean RT for all trials will still undergo a significant shift. Therefore, looking only at group means is insufficient to fully discriminate between the effects of both models.

\section{Task-dependent differences in model sensitivity}

While the simulations summarized above demonstrate that an urgency-gating model can indeed reproduce the results of Winkel et al.'s behavioral data, one final apparent discrepancy remains. In our previous experiments using discrete evidence (Cisek, Puskas, \& El-Murr, 2009) or random-dot motion (Thura et al., 2012) we presented biases in early evidence lasting up to $1000 \mathrm{~ms}$, and these had no significant effect on RTs for decisions made after these biases (RT $>1000 \mathrm{~ms}$ ). Therefore, one must ask: how could a short bias (as in Winkel et al.'s experiment) have a detectable effect on RTs while long ones (as in our previous experiments) do not?

One possibility is that the filter time-constant is contextdependent (Ghose, 2006; Ossmy et al., 2013). While that is a viable possibility, we believe a simpler answer is that what changes depending on task-context is the mean rate at which the urgency signal grows over time (i.e., the steepness of its slope). In a task where success rates asymptote after a short observation time, further observation only reduces reward rates (Thura et al., 2012). Because reward rate is often optimized at the cost of accuracy (Balci et al., 2011), subjects in such circumstances ought to adopt a steep urgency signal in order to make decisions more quickly. The classic random-dot motion task is of this sort, and the urgency signal we use here to simulate it tends to be relatively steep, producing mean RTs around $1200 \mathrm{~ms}$ - about $25 \%$ of which are shorter than $1000 \mathrm{~ms}$. In that distribution of RTs, a significant portion of the earliest reactions will occur before the early stimulus information has leaked out (see the grey lines in Fig. 5), and this subset of early decision times will cause a significant change in the overall mean RT.

However, for tasks in which success rates do not asymptote as rapidly - or when evidence can change - it can be worthwhile to wait longer to see if evidence improves (see Thura et al., 2012, for a derivation of the theoretically optimum policy). Indeed, in contrast to Winkel et al.'s experiment, raw RTs under such conditions in our previous studies were almost never below $1 \mathrm{~s}$ and tended to have means of approximately $3 \mathrm{~s}$. To simulate such results with an urgency-gating model, a shallower urgency signal is needed. Consequently, under such conditions, there are almost never any RTs so fast that the

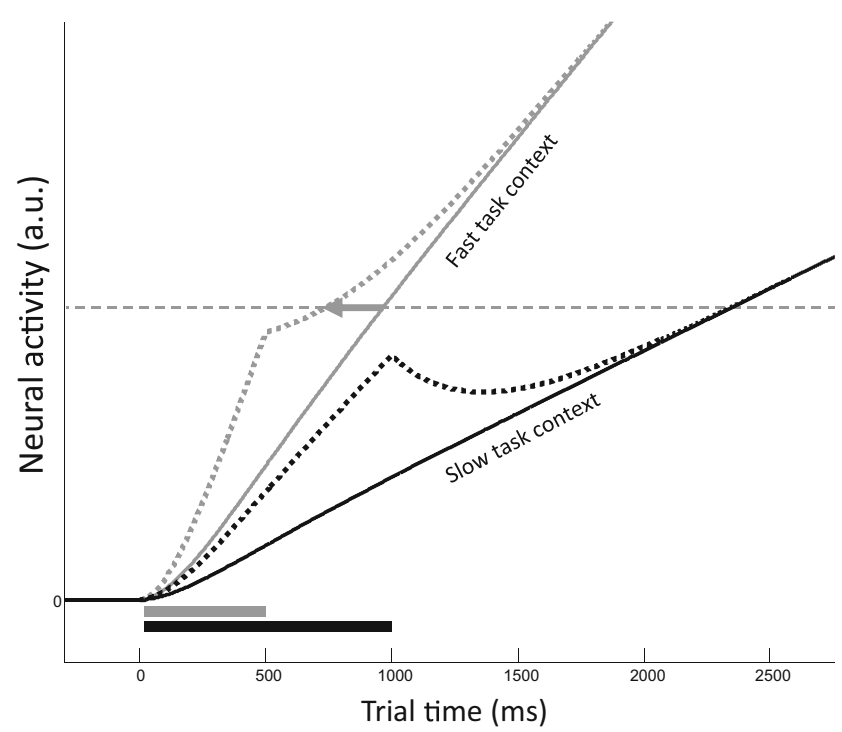

Fig. 5 Simulated neural activity in two different task contexts with identical baseline evidence strength. In the "fast" context, the urgency signal's average slope tends to be high, reflecting a task in which a speedier response policy tends to be advantageous. The solid grey line portrays a trial in which the evidence remains at a single fixed level throughout the entire trial; the dotted line depicts a trial with an early bias during which the evidence is briefly doubled for $500 \mathrm{~ms}$, after which evidence strength drops to the same (baseline) level as the constant-evidence trial. In the "slow" condition, the urgency signal's mean slope is substantially lower, reflecting a task context in which a slower response policy is more advantageous. The solid black line depicts a constant-evidence trial whose evidence strength is identical to that in the "fast" context, but which reaches a fixed threshold substantially later in the trial due to the shallower urgency signal. The dotted black line illustrates a trial with an early bias lasting $1000 \mathrm{~ms}$ in duration. The dashed horizontal line represents a single neural activity threshold, producing distributions of response times whose means will be $\sim 1000 \mathrm{~ms}$ in the "fast" context and $2250 \mathrm{~ms}$ in the "slow" context. Note that, in spite of its much longer duration, the 1000-ms bias in the "slow" condition should not substantially affect the response times in this context, as the bias will have fully leaked away by the time the threshold is reached. In contrast, an early bias only $500 \mathrm{~ms}$ in duration will affect response times in the "fast" context, and these bias trials may therefore skew the group mean reaction time (RT) for all trials in this task condition 
evidence has not had the time to leak out (see the black lines in Fig. 5), and RTs therefore show little or no difference of the early bias.

We have further substantiated this hypothesis in a recent experiment with human subjects, so far only available in abstract form (Carland et al., 2013). In these experiments, we use the random-dot motion task with brief motion pulses, and compare RTs for identical trials in two separate conditions; one in which subjects are motivated to respond quickly (steep urgency), versus one where they respond more slowly (shallow urgency). In the former condition, RTs are significantly influenced by early motion pulses but not by late ones, in a manner consistent with a low-pass filter whose time constant is fairly short. This agrees with previous studies (Huk \& Shadlen, 2005; Kiani, Hanks, \& Shadlen, 2008), which found that only early pulses $(<300 \mathrm{~ms})$ were effective in affecting RTs. Importantly, however, in our latter condition in which responses to identical trials are slower (presumably owing to a shallower urgency signal), RTs are no longer affected by those same early pulses but are instead affected by late pulses. This is consistent with a leaky evidence signal whose activity is governed by a low-pass filter with a short time constant, but is not compatible with any model that does not include a strong leak term.

\section{Conclusion}

While Winkel et al. (2014) designed and conducted a very interesting study that aimed to distinguish between the driftdiffusion model and the urgency-gating model, they failed to consider two important points. First, they did not implement the UGM correctly because they left out a crucial piece - the low-pass filter, which is necessary whenever significant noise is present in the input signal (Cisek et al., 2009). Second, they dismissed the possibility of a strong leak because they focused only on the means of their RT distributions. Here, we show that if the UGM is implemented properly with a low-pass filter, then it successfully captures the behavioral data reported by Winkel et al., and does indeed explain the effects of early evidence.

Acknowledgments We thank the reviewers and the editor for constructive suggestions, and Winkel et al. for making their experimental data available. This work was supported by operating grants from the Natural Sciences and Engineering Research Council (NSERC, \#327654) and the Canadian Institutes of Health Research (CIHR, \#MOP-102662).

\section{References}

Balci, F., Simen, P., Niyogi, R., Saxe, A., Hughes, J. A., Holmes, P., \& Cohen, J. D. (2011). Acquisition of decision making criteria:
Reward rate ultimately beats accuracy. Attention Perception \& Psychopysics, 73, 640-657.

Bogacz, R., Brown, E., Moehlis, J., Holmes, P., \& Cohen, J. D. (2006). The physics of optimal decision making: A formal analysis of models of performance in two-alternative forced-choice tasks. Psychology Review, 113, 700-765.

Busemeyer, J. R., \& Townsend, J. T. (1993). Decision field theory: A dynamic-cognitive approach to decision making in an uncertain environment. Psychology Review, 100, 432-459.

Carland, M., Marcos, E., Thura, D., Verschure, P., \& Cisek, P. (2013). Decision-making is influenced by a context-dependent urgency signal. Program No.286.11.2013 Neuroscience Meeting Planner, San Diego, CA: Society for Neuroscience. Online.

Carpenter, R. H., \& Williams, M. L. (1995). Neural computation of log likelihood in control of saccadic eye movements. Nature, 377, 5962.

Churchland, A. K., Kiani, R., \& Shadlen, M. N. (2008). Decision-making with multiple alternatives. Nature Neuroscience, 11, 693-702.

Cisek, P., Puskas, G. A., \& El-Murr, S. (2009). Decisions in changing conditions: The urgency-gating model. Journal of Neuroscience, 29, $11560-11571$.

Ditterich, J. (2006). Evidence for time-variant decision making. European Journal of Neuroscience, 24, 3628-3641.

Domenech, P., \& Dreher, J. C. (2010). Decision threshold modulation in the human brain. Journal of Neuroscience, 30, 14305-14317.

Ghose, G. M. (2006). Strategies optimize the detection of motion transients. Journal of Vision, 6, 429-440.

Gluth, S., Rieskamp, J., \& Buchel, C. (2012). Deciding when to decide: Time-variant sequential sampling models explain the emergence of value-based decisions in the human brain. Journal of Neuroscience, 32, 10686-10698.

Gold, J. I., \& Shadlen, M. N. (2007). The neural basis of decision making. Annual Review of Neuroscience, 30, 535-574.

Heekeren, H. R., Marrett, S., \& Ungerleider, L. G. (2008). The neural systems that mediate human perceptual decision making. Nature Reviews Neuroscience, 9, 467-479.

Huk, A. C., \& Shadlen, M. N. (2005). Neural activity in macaque parietal cortex reflects temporal integration of visual motion signals during perceptual decision making. Journal of Neuroscience, 25, 1042010436.

Kiani, R., Hanks, T. D., \& Shadlen, M. N. (2008). Bounded integration in parietal cortex underlies decisions even when viewing duration is dictated by the environment. Journal of Neuroscience, 28, 30173029.

Ludwig, C. J., Gilchrist, I. D., McSorley, E., \& Baddeley, R. J. (2005). The temporal impulse response underlying saccadic decisions. Journal of Neuroscience, 25, 9907-9912.

Ossmy, O., Moran, R., Pfeffer, T., Tsetsos, K., Usher, M., \& Donner, T. H. (2013). The timescale of perceptual evidence integration can be adapted to the environment. Current Biology, 23, 981-986.

Palmer, J., Huk, A. C., \& Shadlen, M. N. (2005). The effect of stimulus strength on the speed and accuracy of a perceptual decision. Journal of Vision, 5, 376-404.

Ratcliff, R. (1978). A theory of memory retrieval. Psychological Review, $83,59-108$.

Ratcliff, R., Hasegawa, Y. T., Hasegawa, R. P., Smith, P. L., \& Segraves, M. A. (2007). Dual diffusion model for single-cell recording data from the superior colliculus in a brightness-discrimination task. Journal of Neurophysiology, 97, 1756-1774.

Roitman, J. D., \& Shadlen, M. N. (2002). Response of neurons in the lateral intraparietal area during a combined visual discrimination reaction time task. Journal of Neuroscience, 22, 9475-9489.

Thura, D., Beauregard-Racine, J., Fradet, C. W., \& Cisek, P. (2012). Decision making by urgency gating: Theory and experimental support. Journal of Neurophysiology, 108, 2912-2930. 
Thura, D., \& Cisek, P. (2014). Deliberation and commitment in the premotor and primary motor cortex during dynamic decision-making. Neuron, 81, 1401-1416.

Thura, D., Cos, I., Trung, J., \& Cisek, P. (2014). Context-dependent urgency influences speed-accuracy trade-offs in decision-making and movement execution. Journal of Neuroscience, 34, 1644216454.
Usher, M., \& McClelland, J. L. (2001). The time course of perceptual choice: The leaky, competing accumulator model. Psychology Review, 108, 550-592.

Winkel, J., Keuken, M. C., van Maanen, L., Wagenmakers, E. J., \& Forstmann, B. U. (2014). Early evidence affects later decisions: Why evidence accumulation is required to explain response time data. Psychonomics Bulletin and Review, 21, 777-784. 